\title{
Magneto-orbital texture in the perovskite modification of $\mathrm{Mn}_{2} \mathrm{O}_{3}$
}

\author{
Dmitry D. Khalyavin, ${ }^{1, *}$ Roger D. Johnson, ${ }^{2}$ Pascal Manuel, ${ }^{1}$ Alexander A. Tsirlin, ${ }^{3}$ Artem M. \\ Abakumov, ${ }^{4}$ Denis P. Kozlenko, ${ }^{5}$ Young Sun, ${ }^{6}$ Leonid Dubrovinsky, ${ }^{7}$ and Sergey V. Ovsyannikov ${ }^{7,8}$ \\ ${ }^{1}$ ISIS facility, STFC, Rutherford Appleton Laboratory, Chilton, Didcot, Oxfordshire, OX11-0QX,UK \\ ${ }^{2}$ Department of Physics, Clarendon Laboratory, University of Oxford, Oxford OX1 3PU, United Kingdom \\ ${ }^{3}$ Experimental Physics VI, Center for Electronic Correlations and Magnetism, \\ Institute of Physics, University of Augsburg, 86135 Augsburg, Germany \\ ${ }^{4}$ Center for Electrochemical Energy Storage, Skolkovo Institute of \\ Science and Technology, Nobel Street 3, 143026 Moscow, Russia \\ ${ }^{5}$ Frank Laboratory of Neutron Physics, Joint Institute for Nuclear Research, 141980 Dubna, Russia \\ ${ }^{6}$ Beijing National Laboratory for Condensed Matter Physics, \\ Institute of Physics, Chinese Academy of Sciences, Beijing 100190, China \\ ${ }^{7}$ Bayerisches Geoinstitut, Universität Bayreuth, Universitätsstrasse 30, 95447 Bayreuth, Germany \\ ${ }^{8}$ Institute for Solid State Chemistry, Russian Academy of Sciences, \\ Urals Division, 91 Pervomayskaya Str., Yekaterinburg 620990, Russia
}

(Dated: May 2, 2018)

\begin{abstract}
Crystal and magnetic structures of the high-pressure-stabilized perovskite modification of $\mathrm{Mn}_{2} \mathrm{O}_{3}\left(\zeta-\mathrm{Mn}_{2} \mathrm{O}_{3}\right)$ have been studied by neutron powder diffraction combined with symmetry arguments based on the phenomenological Landau theory. This metastable phase exhibits a unique charge disproportionation phenomenon stabilizing the quadruple perovskite structure $\left(\mathrm{Mn}^{2+} \mathrm{Mn}_{3}^{3+}\right) \mathrm{Mn}_{4}^{3.25+} \mathrm{O}_{12}$ with an additional charge-ordering and commensurate orbital density wave localized in the B-site perovskite position. The commensurate nature of the orbital density wave is stimulated by a coupling of the orbital ordering to independent structural distortions, which improve poor bonding conditions of $\mathrm{Mn}^{2+}$ in the A-site perovskite position. Below $T_{1} \sim 100 \mathrm{~K}$, an anharmonic longitudinal spin density wave arises and locks to the structural modulation associated with the orbital density. At $T_{2} \sim 50 \mathrm{~K}$, the magnetic subsystem de-locks from the structural modulation giving rise to a multi- $k$ phase-modulated ground state admixing cycloidal and helical components. The complex anharmonic and phase-modulated magnetic structures are discussed based on a phenomenological magneto-orbital coupling scheme, previously developed to explain the multi- $k$ helical ground states with modulated spin chirality observed in $\mathrm{A}^{2+} \mathrm{Mn}_{7} \mathrm{O}_{12}\left(\mathrm{~A}^{2+}=\mathrm{Ca}, \mathrm{Sr}, \mathrm{Pb}\right.$ and $\left.\mathrm{Cd}\right)$ quadruple perovskites.
\end{abstract}

PACS numbers: 75.10.-b, 75.25.-j, 75.25.Dk

\section{INTRODUCTION}

Quadruple perovskite manganites of the family $\left(\mathrm{A}^{2+} \mathrm{Mn}_{3}^{3+}\right) \mathrm{Mn}_{4}^{3.25+} \mathrm{O}_{12}\left(\mathrm{~A}^{2+}=\mathrm{Ca}, \mathrm{Sr}, \mathrm{Pb}\right.$ and $\left.\mathrm{Cd}\right)$ provide a unique playground to study interplay between charge, spin, and orbital degrees of freedom [1-13]. The prototype compound $\mathrm{CaMn}_{7} \mathrm{O}_{12}$ has been shown to exhibit 3:1 charge order between $\mathrm{Mn}^{3+}$ and $\mathrm{Mn}^{4+}$ in the B-site perovskite position [3, 4], which occurs well above room temperature at $T_{\mathrm{CO}} \sim 409 \mathrm{~K}$. At lower temperature, $T_{\mathrm{OO}} \sim 260 \mathrm{~K}$, incommensurate orbital ordering (orbital density wave) takes place $[1,4-6]$ followed by the onset of long-range magnetic order at $T_{1} \sim 90 \mathrm{~K}$ $[2,5,6]$. The fundamental magnetic propagation vector, $\boldsymbol{k}_{\mathbf{0}}$, is locked to the structural modulation, $\boldsymbol{k}_{\boldsymbol{S}}$, associated with the orbital density wave through the relation $\boldsymbol{k}_{0}=\boldsymbol{k}_{\boldsymbol{S}} / 2[2,5,6]$. At a critical temperature $T_{2} \sim$ $48 \mathrm{~K}$, the magnetic subsystem de-locks from the structural modulation, resulting in an unprecedentedly complex multi- $k$ magnetic ground state $[7,8]$ with prominent multiferroic properties $[2,9,10]$. The corresponding magnetic structure is a nearly constant moment helix with incommensurately modulated spin chirality [8].
This type of magnetic structure is stabilized by a set of competing exchange interactions and anisotropies modulated by the orbital density wave. The respective coupling phenomenon, which is responsible for the admixture of distinct magnetic order parameters that give rise to the incommensurate modulation of the spin chirality, was referred to as magneto-orbital coupling $[1,8]$. The other members of the $\mathrm{A}^{2+} \mathrm{Mn}_{7} \mathrm{O}_{12}$ family exhibit qualitatively similar behaviour with the corresponding modulations and the critical temperatures being dependent on the nature of the di-valent A-site cation [11-13].

Recently, it has been shown that at high temperature (above $T \sim 1000 \mathrm{~K}$ ) and at high-pressure (above $P \sim$ $18 \mathrm{GPa}$ ), $\mathrm{Mn}_{2} \mathrm{O}_{3}$ transforms to a perovskite modification, denoted as $\zeta$-polymorph, which is quenchable as a metastable phase at ambient conditions [14]. This exotic modification of $\mathrm{Mn}_{2} \mathrm{O}_{3}$ falls into the same family of quadruple perovskites discussed above, where the divalent A-site is taken by $\mathrm{Mn}^{2+}$ cations, and exhibits multiferroic properties tuneable by the magnetic field [15]. The room-temperature diffraction studies [13] revealed that, to a good approximation, the structure is trigonal with the space group $R \overline{3}$. This observation again points 
to the $3: 1 \mathrm{~B}$-site $\mathrm{Mn}^{3+} / \mathrm{Mn}^{4+}$ charge ordering, which is a precursor for the orbital density wave formation that promotes the rich magneto-orbital physics.

In this paper, we study in detail the interplay between the structural and magnetic subsystems of the $\zeta-\mathrm{Mn}_{2} \mathrm{O}_{3}$ compound by means of neutron powder diffraction. The obtained results reveal that our $\zeta-\mathrm{Mn}_{2} \mathrm{O}_{3}$ sample exhibits a commensurate orbital density wave and two distinct magnetic phases. The high-temperature phase is an anharmonic spin density wave locked to the structural modulation associated with the orbital ordering, and the low-temperature magnetic structure is a complex phasemodulated cycloid with a small admixture of a helical component. Hence, the models developed for the description of similar magneto-orbital coupling in the $\mathrm{AMn}_{7} \mathrm{O}_{12}$ family were extended for the present case, where the Asite sublattice is magnetic as well. The crystal and magnetic structures of $\zeta-\mathrm{Mn}_{2} \mathrm{O}_{3}$ confirm universality of the magneto-orbital coupling in the divalent A-site quadruple perovskite manganites, both in the formation and the evolution of the complex magnetic ground state.

\section{EXPERIMENTAL SECTION}

Samples of $\zeta-\mathrm{Mn}_{2} \mathrm{O}_{3}$ were synthesized from a commercial powder of the conventional cubic-bixbyite-structured $\mathrm{Mn}_{2} \mathrm{O}_{3}$ (from Chempur company, with $99.99 \%$ chemical purity) at high-pressure high-temperature conditions. These syntheses were accomplished using the multi-tonne multi-anvil presses at BGI (Bayreuth, Germany) at a pressure of $19 \mathrm{GPa}$ and at $900{ }^{\circ} \mathrm{C}$, lying within the stability region of this perovskite-type polymorph previously established [14]. We used a standard assembly for these syntheses, which included a Re cylindrical sample capsule, a $\mathrm{LaCrO}_{3}$ heater, W3Re/W25Re thermocouples, and an octahedral container, similar to that reported in our previous work [16]. Typical synthesis times were approximately $10 \mathrm{~h}$ or longer to ensure a complete transformation of the sample to the perovskite-type polymorph. We analysed the recovered products of the syntheses by conventional chemical and structural methods, and selected several high-quality polycrystalline samples of the perovskite phase for the present neutron diffraction study.

The neutron powder diffraction data were collected at the ISIS pulsed neutron and muon facility of the Rutherford Appleton Laboratory (UK) on the WISH diffractometer [17] and at the IBR-2 high-flux pulsed reactor (FNLP Dubna, Russia) on the DN-12 diffractometer [18]. In both cases, samples $(\sim 40 \mathrm{mg})$ were loaded into cylindrical vanadium cans and measured in the temperature range of 1.5-120 K using an Oxford Instrument Cryostat (WISH data) and 10-200 K using a He-closed cycle refrigerator (DN-12 data). Rietveld refinements of the crystal and magnetic structures were performed using the Full-

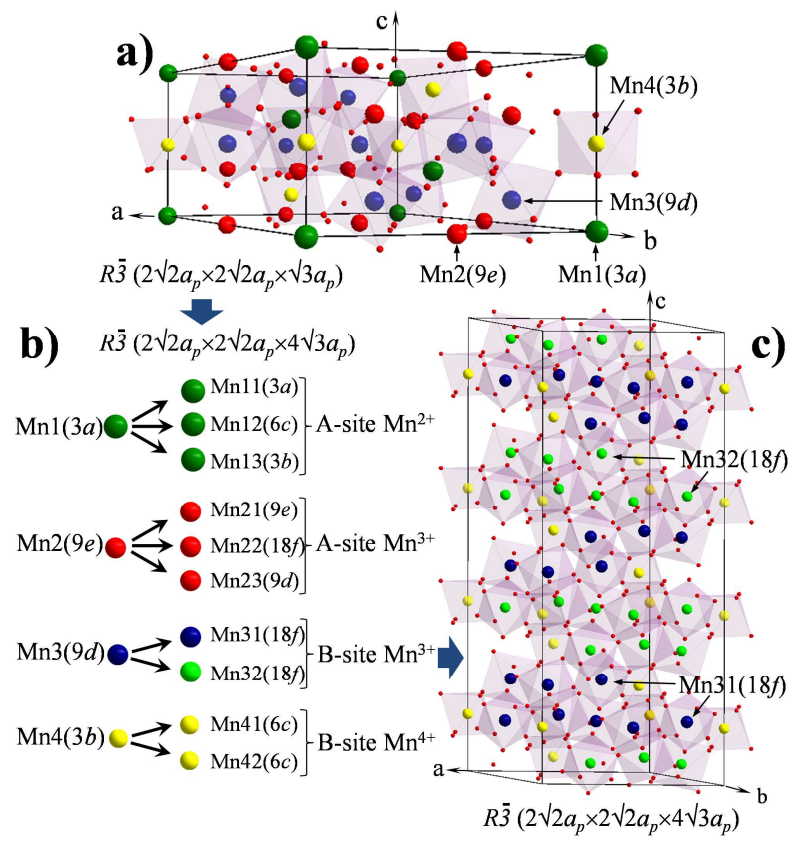

FIG. 1. (a) Schematic representation of the parent $R \overline{3}$ $\left(2 \sqrt{2} a_{p} \times 2 \sqrt{2} a_{p} \times \sqrt{3} a_{p}\right)$ crystal structure used in the analysis of orbital and magnetic orderings of $\zeta-\mathrm{Mn}_{2} \mathrm{O}_{3}$. Mn takes four symmetry inequivalent sites: Mn1 $[3 a(0,0,0)]$, Mn2 $[9 e(0.5,0,0)], \operatorname{Mn} 3[9 d(0.5,0,0,5)]$, and Mn4 $[3 b(0,0,0.5)]$. (b) Wyckoff position splitting at the transition from $R \overline{3}$ $\left(2 \sqrt{2} a_{p} \times 2 \sqrt{2} a_{p} \times \sqrt{3} a_{p}\right)$ to $R \overline{3}\left(2 \sqrt{2} a_{p} \times 2 \sqrt{2} a_{p} \times 4 \sqrt{3} a_{p}\right)$ crystal structures. (c) Schematic representation of the $R \overline{3}$ $\left(2 \sqrt{2} a_{p} \times 2 \sqrt{2} a_{p} \times 4 \sqrt{3} a_{p}\right)$ crystal structure, demonstrating the Wyckoff position splitting for the B-site $\mathrm{Mn}^{3+}$ (A-site Mn is omitted for clarity).

prof program [19] against the data measured in detector banks at average $2 \theta$ values of $58^{\circ}, 90^{\circ}, 122^{\circ}$, and $154^{\circ}$ (WISH instrument) and $45.5^{\circ}$ and $90^{\circ}$ (DN-12 instrument).

\section{RESULTS}

\section{Crystal structure and orbital ordering}

The crystal structure of the $\zeta$-modification of $\mathrm{Mn}_{2} \mathrm{O}_{3}$ has been reported to be triclinic with a complex commensurate superstructure that quadruples the pseudocubic unit cell in all three directions [14]. The triclinic distortion, however, was found to be very small and the full structure can be considered as trigonal to a good approximation (deviations of the unit cell parameters from the trigonal metric are less than $0.1 \%$ ). To understand the nature of the superstructure and reveal the interplay between different distortions involved, we analysed the high-resolution neutron diffraction data using comprehensive symmetry arguments.

A neutron diffraction pattern in the paramagnetic 
phase of $\zeta-\mathrm{Mn}_{2} \mathrm{O}_{3}$ was collected at $T=120 \mathrm{~K}$. Splitting of the fundamental reflections, consistent with the rhombohedral metric of the pseudocubic perovskite unit cell, and the presence of superstructure reflections, which double the cell in two directions, both resemble the average $R \overline{3}\left(2 \sqrt{2} a_{p} \times 2 \sqrt{2} a_{p} \times \sqrt{3} a_{p}\right)$ structure of $\mathrm{CaMn}_{7} \mathrm{O}_{12}$. The small triclinic distortion is beyond the resolution limit of the present diffraction experiment and will be not considered in the subsequent analysis. The trigonal structure involves two types of primary distortions, namely, the octahedral tilting $\left(a^{+} a^{+} a^{+}\right)$ and 3:1 $\mathrm{Mn}^{3+} / \mathrm{Mn}^{4+}$ charge ordering in the B-site perovskite position (see Fig. 1a). We will use this $R \overline{3}\left(2 \sqrt{2} a_{p} \times 2 \sqrt{2} a_{p} \times \sqrt{3} a_{p}\right)$ symmetry as the parent one to discuss additional structural distortions and the magnetic ordering in the system. This space group is adapted for the sake of simplicity as a sufficiently "minimal" parent symmetry to classify relevant order parameters and describe coupling between them (note that transition to a phase with this symmetry need not actually exist in $\left.\zeta-\mathrm{Mn}_{2} \mathrm{O}_{3}\right)$. We could equally use the cubic perovskite $\operatorname{Pm} \overline{3} m$ space group as the parent one, but this would result in an unnecessarily complicated form of the relevant free-energy coupling terms.

The $\left(2 \sqrt{2} a_{p} \times 2 \sqrt{2} a_{p} \times \sqrt{3} a_{p}\right)$ unit cell does not account for a set of rather strong reflections (Fig. 2a), which require a further doubling of the cell along the $c$-direction (propagation vector $\left.\boldsymbol{k}_{\boldsymbol{T}}=(0,0,3 / 2)\right)$. Two isotropy subgroups associated with the one-dimensional $\mathrm{T}_{1}^{+}(\varepsilon)$ and $\mathrm{T}_{1}^{-}(\varepsilon)$ irreducible representations (irreps) related to $\boldsymbol{k}_{\boldsymbol{T}}$ preserve the three-fold symmetry [20, 21], and therefore were tested in the refinement procedure. Both irreps break only translational symmetry resulting in the $R \overline{3}$ subgroup with the unit cell doubled along the $c$-axis. The difference between $\mathrm{T}_{1}^{+}(\varepsilon)$ and $\mathrm{T}_{1}^{-}(\varepsilon)$ is in the origin choice, being at $(0,0,0)$ and $(0,0,1 / 2)$, respectively. The refinement unambiguously yields the distortion of the $\mathrm{T}_{1}^{+}(\varepsilon)$ irrep as the relevant order parameter. The oxygen displacement modes with the $\mathrm{T}_{1}^{+}$symmetry have largest amplitudes and a portion of these displacements is shown in Figure 2c (inset). It is clear that these distortions serve to improve a poor bonding condition of $\mathrm{Mn}^{2+}$, which is otherwise too small for the A-site perovskite position ( $3 a$ site in the parent structure, see Fig. 1a). The presence of these displacement modes indeed improves the average bond valence sums (BVS) of the $3 a$ Mn-site from 1.2 to 1.8 valence units. The $\mathrm{T}_{1}^{+}$distortions are large, indicating that they are a crucial ingredient stabilising the structure and probably exist in the whole region of stability of the perovskite phase.

In the original work mentioned above [14], electron diffraction was used to show that the perovskite structure of $\zeta-\mathrm{Mn}_{2} \mathrm{O}_{3}$ also involves a distortion that quadruples the pseudocubic perovskite cell in all three direc-
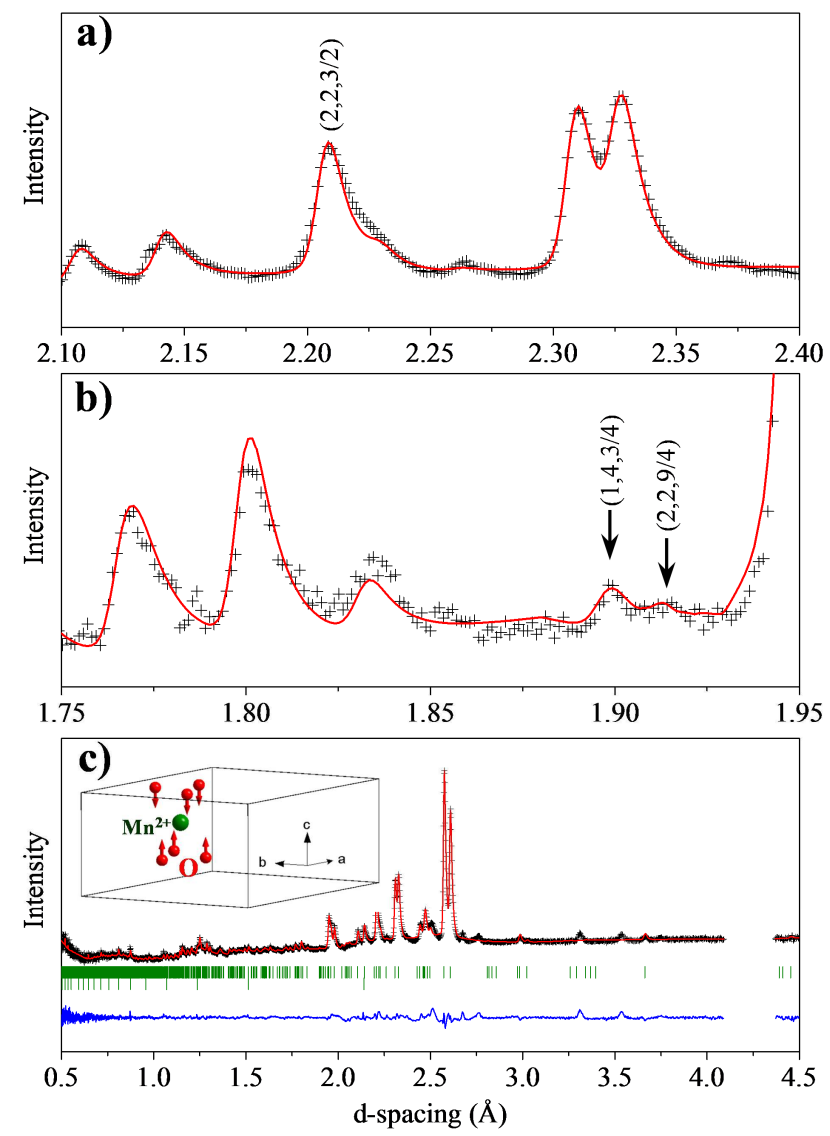

FIG. 2. Neutron diffraction pattern $(T=120 \mathrm{~K})$ in the vicinity of the superstructure reflections associated with $\boldsymbol{k}_{T}=$ $(0,0,3 / 2)$ (a) and $\boldsymbol{k}_{S}=(0,0,3 / 4)$ (b) propagation vectors. (c) Rietveld refinement of the pattern collected at the highresolution backscattering detector bank and using the $R \overline{3}$ $\left(2 \sqrt{2} a_{p} \times 2 \sqrt{2} a_{p} \times \sqrt{3} a_{p}\right)$ structural model. The cross symbols (black) and solid line (red) represent the experimental and calculated intensities, respectively, and the line below (blue) is the difference between them. Tick marks indicate the positions of Bragg peaks for $\zeta-\mathrm{Mn}_{2} \mathrm{O}_{3}$ (top) and vanadium can (bottom). The inset shows a portion of T-displacement mode serving to improve bonding conditions of A-site $\mathrm{Mn}^{2+}$ cation.

tions. The relevant propagation vector is $\boldsymbol{k}_{S}=(0,0,3 / 4)$ in the setting of the $R \overline{3}\left(2 \sqrt{2} a_{p} \times 2 \sqrt{2} a_{p} \times \sqrt{3} a_{p}\right)$ space group ( $\Lambda$-line of symmetry). Indeed, very weak reflections indexed by this propagation vector can be found in the neutron diffraction pattern (Fig. 2b). In general, by combining the distortions with the $\boldsymbol{k}_{\boldsymbol{T}}$ and $\boldsymbol{k}_{\boldsymbol{S}} \bmod -$ ulations, two qualitatively distinct situations are possible, depending on weather these distortions are coupled by symmetry or not: i) the order parameters associated with both propagation vectors are decoupled and therefore the symmetry is defined by their intersection $T \oplus \Lambda$, ii) the order parameters are coupled and the distortion associated with $\boldsymbol{k}_{\boldsymbol{S}}$ is sufficient to define the final symmetry of the system. In the latter case, the $\mathrm{T}_{1}^{+}(\varepsilon)$ distortion modes have the symmetry of a secondary order param- 
TABLE I. Table I. Isotropy subgroups associated with the $\Lambda_{1}$ irrep and $\boldsymbol{k}_{\boldsymbol{S}}=(0,0,3 / 4)$ propagation vector of the parent group $R \overline{3}\left(2 \sqrt{2} a_{p} \times 2 \sqrt{2} a_{p} \times \sqrt{3} a_{p}\right)$. The table provides the space group symmetry, order parameter direction, basis vectors and origin of the subgroups in respect of the parent structure.

\begin{tabular}{llll}
\hline \hline SG & OP & Basis & Origin \\
\hline$R \overline{3}$ & $(\delta, 0)$ & $(-1,-1,0),(1,0,0),(0,0,4)$ & $(0,0,0)$ \\
$R \overline{3}$ & $(\delta,-\delta)$ & $(-1,-1,0),(1,0,0),(0,0,4)$ & $(0,0,3 / 2)$ \\
$R \overline{3}$ & $\left(\delta_{1}, \delta_{2}\right)$ & $(-1,-1,0),(1,0,0),(0,0,4)$ & $(0,0,0)$ \\
\hline \hline
\end{tabular}

eter (in the sense of their symmetry compatibility with the $\Lambda$-distortion). Note, the situation when there is coupling and the symmetry is defined by the $\boldsymbol{k}_{T}$ order parameter is impossible due to constraints imposed by the translational symmetry. The first scenario necessarily requires a violation of $R$-centring, which is inconsistent with the present neutron diffraction data as well as with the electron diffraction reported in Ref. [14]. In the second scenario, one can distinguish three main candidates for the final symmetry of $\zeta-\mathrm{Mn}_{2} \mathrm{O}_{3}$ by inspecting all possible isotropy subgroups related to the $\boldsymbol{k}_{\boldsymbol{S}}=(0,0,3 / 4)$ propagation vector $[20,21]$. They maintain the $R$-centred lattice and they are associated with the two-dimensional $\Lambda_{1}\left(\delta_{1}, \delta_{2}\right)$ irrep. The relevant order parameter directions, lattice vectors, and origins, with respect to the parent $R \overline{3}$ $\left(2 \sqrt{2} a_{p} \times 2 \sqrt{2} a_{p} \times \sqrt{3} a_{p}\right)$ structure are given in Table I. The three subgroups correspond to different choices of the global phase of the structural modulation being 0 , $\pi / 4$, and an arbitrary phase (different from 0 and $\pi / 4$ ), respectively. The final choice can be done by applying the above-discussed criterion that the symmetry should be compatible with the $\mathrm{T}_{1}^{+}(\varepsilon)$ distortion modes (should allow coupling between the $\mathrm{T}_{1}^{+}$and $\Lambda_{1}$ distortion modes). This criterion can be verified using the appropriate freeenergy term which couples the $\Lambda_{1}\left(\delta_{1}, \delta_{2}\right)$ and $\mathrm{T}_{1}^{+}(\varepsilon)$ order parameters [22]. The general form of the lowest degree free-energy invariant is $\varepsilon \delta_{1}^{2}-\varepsilon \delta_{2}^{2}$. The term does not vanish for the first and the third cases, but the coupling is maximal for the former case, making the $\Lambda_{1}(\delta, 0)$ candidate with the $R \overline{3}$ symmetry and the origin at $(0,0,0)$, the most appropriate one. Note that this structural model is identical to the one given in the original paper [14]. The final refinement using this symmetry resulted in a good agreement with the experimental data (Fig. 2c) and the atomic coordinates are given in Table $1 \mathrm{~S}$ of the Supplemental Material [23].

The structural model with the $\left(2 \sqrt{2} a_{p} \times 2 \sqrt{2} a_{p} \times\right.$ $\left.4 \sqrt{3} a_{p}\right)$ supercell implies a splitting of both Mn3 (9d) and Mn4 (3b) Wyckoff positions in the B-site sublattice of the parent $\mathrm{CaMn}_{7} \mathrm{O}_{12}$ structure into two symmetry non-equivalent sites Mn31 (18f), Mn32 (18f) and Mn41 (6c), Mn42 (6c), respectively (see Table $1 \mathrm{~S}$ and Fig.

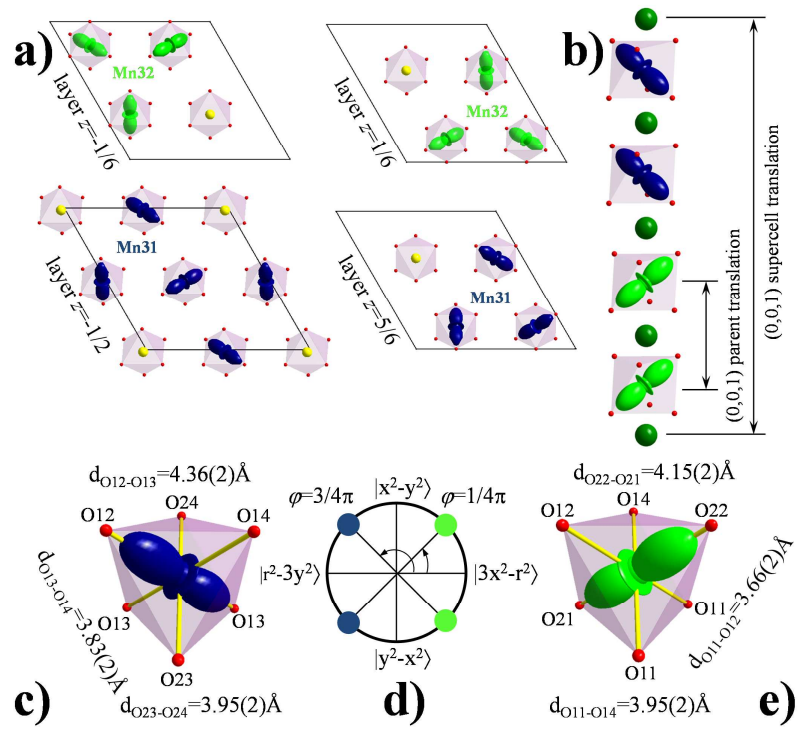

FIG. 3. (a) Four structural layers illustrating the orbital density wave localized on the $\mathrm{B}$-site $\mathrm{Mn}^{3+}$ cations in the metastable perovskite $\zeta-\mathrm{Mn}_{2} \mathrm{O}_{3}$ (only the dominant orbitals with probability of electron localization 0.85 are shown). The $z$-coordinate of each layer is given in respect of the parent cell. The B-site $\mathrm{Mn}^{3+}$ cations with different orbital states corresponding to distinct Wyckoff positions in the $\left(2 \sqrt{2} a_{p} \times 2 \sqrt{2} a_{p} \times 4 \sqrt{3} a_{p}\right)$ supercell (Mn31 and Mn32) are shown in different colours. (b) Change of the dominant orbital state of an isolated $\mathrm{Mn}^{3+} \mathrm{O}_{6}$ octahedron upon $(0,0,1)$ parent translations. The anisotropies of oxygen octahedra coordinating B-site $\mathrm{Mn}^{3+}$ ions in the Mn32 and Mn31 positions, and the associated dominant orbital states, are shown in panels (c) and (e), respectively. (d) Diagram illustrating the relation between the orbital states of the $\mathrm{B}$-site $\mathrm{Mn}^{3+}$ cations and the phase $\phi$ of the commensurate structural modulation.

1b, c). BVS calculations confirmed the presence of the B-site charge order in $\zeta-\mathrm{Mn}_{2} \mathrm{O}_{3}$ with $\mathrm{Mn}^{3+}$ occupying the $18 f$ sites $(\mathrm{Mn} 31, \mathrm{Mn} 32)$ and $\mathrm{Mn}^{4+}$ at the $6 c$ sites (Mn41, Mn42) as follows from Table 2S of the Supplemental Material [23]. This 3:1-type of charge order is similar to the one taking place in other members of the $\mathrm{A}^{2+} \mathrm{Mn}_{7} \mathrm{O}_{12}$ series $[3,4,11-13]$. It has been shown that the charge order promotes oxygen displacements, which are coupled to $x^{2}-y^{2}$ orbital state of the Jahn-Teller active sixfold-coordinated $\mathrm{Mn}^{3+}$ cation [24]. This orbital state does not allow full release of the Jahn-Teller instability [25] and therefore the charge-order driven distortion has been suggested to be the key ingredient for the orbital density wave formation in $\mathrm{CaMn}_{7} \mathrm{O}_{12}[1]$ and other members of the $\mathrm{AMn}_{7} \mathrm{O}_{12}$ family [11]. The structural distortions, assisting the orbital density wave, are associated with the $\Lambda$-line of symmetry in all the cases. Thus, by analogy with these perovskites, one can assume that the instability associated with the $\Lambda$-line of symmetry in $\zeta-\mathrm{Mn}_{2} \mathrm{O}_{3}$ is the orbital density wave too. The Wyckoff position splitting implies a $\Psi^{+} \Psi^{+} \Psi^{-} \Psi^{-}$ 
sequence of the orbital states on B-site $\mathrm{Mn}^{3+}$ ions related by lattice translations of the parent structure along the $c$-axis (Fig. 1c and 3a, b). The signs "+" and "-" indicate distinct orbital states. In other words, the Mn31 (18f) and Mn32 (18f) sites, occupied by $\mathrm{Mn}^{3+}$ cations in the $\left(2 \sqrt{2} a_{p} \times 2 \sqrt{2} a_{p} \times 4 \sqrt{3} a_{p}\right)$ supercell, adopt distinct orbital states. The orbital state $\Psi$ of B-site $\mathrm{Mn}^{3+}$ can be defined as a mixture of the $\psi_{1}$ and $\psi_{2}$ orbitals through the relation: $\Psi=\psi_{1}\left|\cos \left(\pi \phi+\phi_{0}\right)\right|+\psi_{2}\left|\sin \left(\pi \phi+\phi_{0}\right)\right|$, where $\phi=\boldsymbol{k}_{\boldsymbol{S}} \cdot(\boldsymbol{r}+\boldsymbol{t})$ is the phase of the structural modulation, $\boldsymbol{r}$ is the vector defining the position of the B-site $\mathrm{Mn}^{3+}$ cation in the unit cell of the parent structure, $\boldsymbol{t}$ is the lattice translation, and $\phi_{0}$ is the global phase. In the following, we adopt an orbital parametrization similar to the case of $\mathrm{CaMn}_{7} \mathrm{O}_{12}$ [1], i.e. $\psi_{1}=\left|3 x^{2}-r^{2}\right\rangle$ and $\psi_{2}=\left|r^{2}-3 y^{2}\right\rangle$. The structural model with the $\Psi^{+} \Psi^{+} \Psi^{-} \Psi^{-}$sequence fixes the global phase of the structural modulation to be zero, $\phi_{0}=0$ (Fig. $3 \mathrm{~d}$ ), and implies alternation of the states dominated by either $\left|3 x^{2}-r^{2}\right\rangle\left(\Psi^{+}=\cos (\pi / 8) \psi_{1}+\sin (\pi / 8) \psi_{2}\right)$ or $\left|r^{2}-3 y^{2}\right\rangle\left(\Psi^{-}=\cos (3 \pi / 8) \psi_{1}+\sin (3 \pi / 8) \psi_{2}\right)$ orbitals, as shown in Figure $3 \mathrm{a}$ and $\mathrm{b}$ (see also Fig. $1 \mathrm{~S}$ of the Supplemental Material [23]). These expressions indicate that the probability of electron localization on the dominant orbital is $[\cos (\pi / 8)]^{2}=[\sin (3 \pi / 8)]^{2}=0.85$. The quantitative structure refinement confirmed the proposed orbital pattern, which can be also anticipated from the anisotropy of the $\mathrm{Mn}^{3+} \mathrm{O}_{6}$ octahedra that have a clear uniaxial elongation (Fig. 3c, e).

The symmetry consideration presented above provides a deep insight into the structural properties of $\zeta-\mathrm{Mn}_{2} \mathrm{O}_{3}$, revealing interplay between the distinct structural instabilities. The T-displacement modes are involved due to the structural instability imposed by the poor bonding conditions of $\mathrm{Mn}^{2+}$ in the A-site of the perovskite lattice. The presence of these distortions forces another independent electronic instability (orbital density wave) to adopt a symmetry that can be coupled to them. So the T-modes lock the modulation associated with the orbital density wave to the commensurate value $\boldsymbol{k}_{\boldsymbol{S}}=(0,0,3 / 4)$, through the linear quadratic coupling term discussed above. The orbital pattern, in turn, has a strong impact on the magnetic structures discussed in the next sections.

\section{High-temperature commensurate magnetic phase}

Magnetic susceptibility and specific heat measurements reported in Ref. [14] revealed two transitions at $T_{1} \sim 100 \mathrm{~K}$ and $T_{2} \sim 50 \mathrm{~K}$ with a possible third transition at $T_{3} \sim 12 \mathrm{~K}$. Our neutron diffraction data indicate that, similar to other $\mathrm{AMn}_{7} \mathrm{O}_{12}$ quadruple perovskites, two distinct magnetic phases occur in $\zeta-\mathrm{Mn}_{2} \mathrm{O}_{3}$. These phases onset at $T_{1}$ and $T_{2}$, while the anomaly at $T_{3}$ does not correspond to any change in the magnetic ground
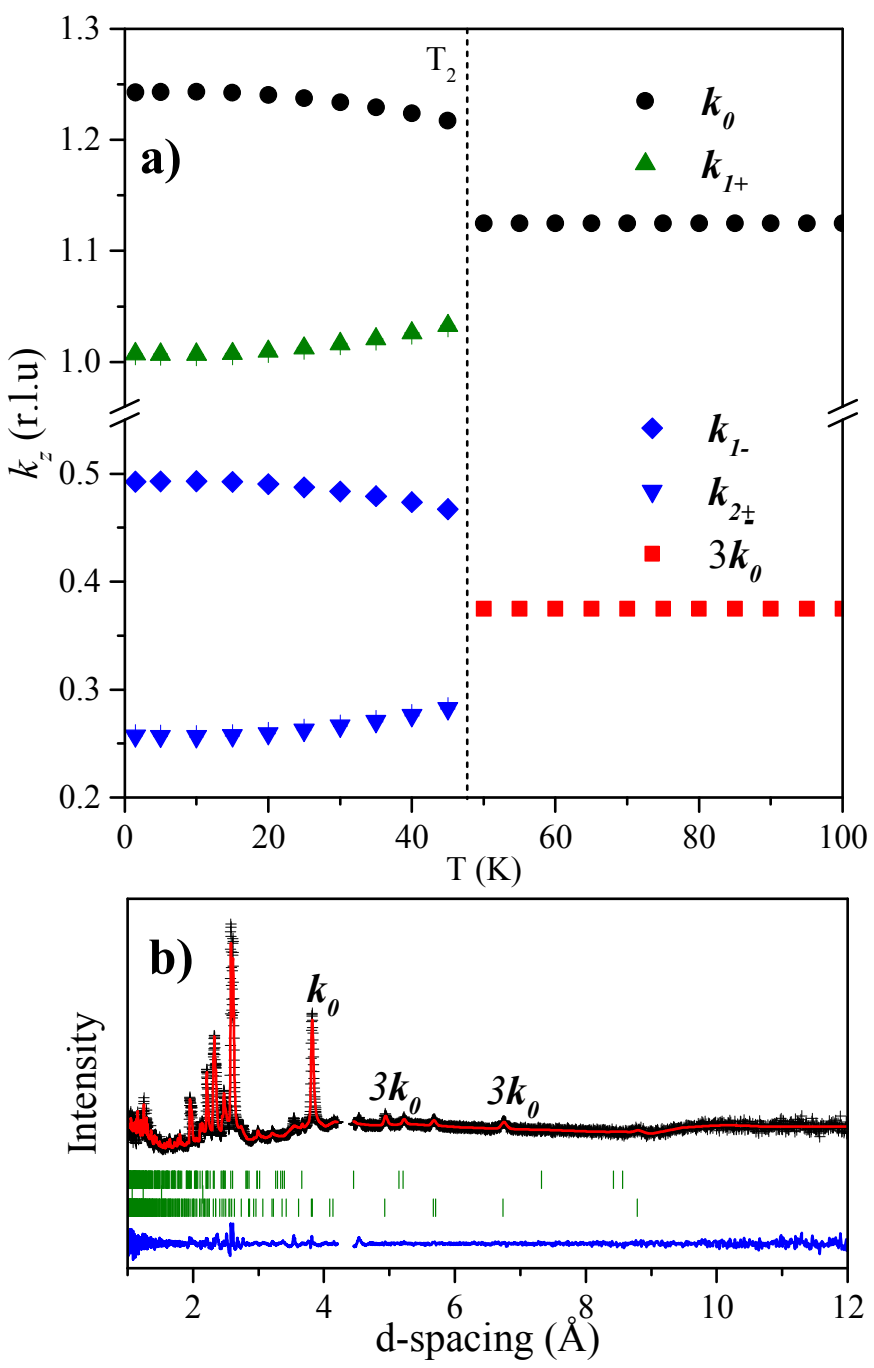

FIG. 4. (a) Temperature variation of the $z$-component of the magnetic propagation vectors taken from the first Brillouin zone. (b) Rietveld refinement of the neutron diffraction pattern collected at $T=55 \mathrm{~K}$. The cross symbols (black) and solid line (red) represent the experimental and calculated intensities, respectively, and the line below (blue) is the difference between them. Tick marks indicate the positions of Bragg peaks for the nuclear (top), vanadium can (middle), and magnetic (bottom) scattering.

state. In the high-temperature phase $\left(T_{1}<T<T_{2}\right)$, the magnetic propagation vector is locked and does not vary with temperature. Below $T_{2}$, it exhibits a substantial temperature evolution, revealing an incommensurate nature of the magnetic ordering (Fig. 4a).

The approach to solve the magnetic structures in $\zeta$ $\mathrm{Mn}_{2} \mathrm{O}_{3}$ is based on the knowledge of the magnetic structures in $\mathrm{AMn}_{7} \mathrm{O}_{12}$ perovskites $[7,11]$. In the latter case, the central role is played by a coupling between an incommensurate orbital-density wave and magnetic order parameters. As discussed above, the orbital density wave is commensurate in $\zeta-\mathrm{Mn}_{2} \mathrm{O}_{3}$, with the propagation vec- 
TABLE II. Magnetic isotropy subgroups associated with the $m \Lambda_{1}$ irrep and $\boldsymbol{k}_{0}=(0,0,9 / 8)$ propagation vector of the parent group $R \overline{3}\left(2 \sqrt{2} a_{p} \times 2 \sqrt{2} a_{p} \times \sqrt{3} a_{p}\right)$. The table provides the space group symmetry, order parameter direction, basis vectors and origin of the subgroups in respect of the parent structure.

\begin{tabular}{llll}
\hline \hline SG & OP & Basis & Origin \\
\hline$R_{I} \overline{3}$ & $(\rho, 0)$ & $(-1,-1,0),(1,0,0),(0,0,8)$ & $(0,0,0)$ \\
$R_{I} \overline{3}$ & $(\rho,[1-\sqrt{2}] \rho)$ & $(-1,-1,0),(1,0,0),(0,0,8)$ & $(0,0,3 / 2)$ \\
$R_{I} 3$ & $\left(\rho_{1}, \rho_{2}\right)$ & $(-1,-1,0),(1,0,0),(0,0,8)$ & $(0,0,0)$ \\
\hline \hline
\end{tabular}

tor $\boldsymbol{k}_{S}=(0,0,3 / 4)$ with respect to the unit cell of the parent $R \overline{3}\left(2 \sqrt{2} a_{p} \times 2 \sqrt{2} a_{p} \times \sqrt{3} a_{p}\right)$ structure. Assuming that the linear-quadratic magneto-elastic coupling is the dominant lock-in term in the free-energy decomposition [7], the propagation vector conservation law requires $\boldsymbol{k}_{\boldsymbol{S}}+2 \boldsymbol{k}_{0}=(0,0,3) n$, where $\boldsymbol{k}_{\mathbf{0}}$ is the fundamental magnetic propagation vector and $n$ is an integer number. This implies that the fundamental magnetic propagation vector is either $\boldsymbol{k}_{\mathbf{0}}=\boldsymbol{k}_{\boldsymbol{S}} / 2=(0,0,3 / 8)$ or $\boldsymbol{k}_{0}=\left[(0,0,3)-\boldsymbol{k}_{\boldsymbol{S}}\right] / 2=(0,0,9 / 8)$. An indexation procedure confirmed that the latter propagation vector can account for the majority of magnetic reflections (including the most intense, fundamental reflections), whilst a few weaker reflections could not be accounted for. However, these weak reflections could be indexed using an additional propagation vector $3 \boldsymbol{k}_{0}=(0,0,3 / 8)$ (Fig. $4 \mathrm{~b}$ ) [26].

An important observation is the absence of $(0,0,3 n) \pm$ $\boldsymbol{k}_{\mathbf{0}}\left(3 \boldsymbol{k}_{\mathbf{0}}\right)$ satellites, which strongly indicates that the moments in the high-temperature phase are oriented along the $c$-axis, giving a longitudinal spin density wave. The next step is to determine the relative magnetic phases of the independent Mn sites. In addition, since the magnetic structure is commensurate, the symmetry of the system depends on the global magnetic phase. There are two time-odd (magnetic) irreducible representations of the $R \overline{3}$ space group associated with the $\Lambda$-line of symmetry, namely, two-dimensional $m \Lambda_{1}\left(\rho_{1}, \rho_{2}\right)$ and fourdimensional $m \Lambda_{2}$. These representations describe different types of magnetic structures [20,21]. Longitudinal spin-density waves are transformed by the $m \Lambda_{1}$ irrep and, therefore, the magnetic structures associated with this representation are the primary candidates for the hightemperature phase of $\zeta-\mathrm{Mn}_{2} \mathrm{O}_{3}$. Depending on the choice of the global phase, there are three symmetry distinct situations as specified in Table II, which correspond to different directions of the magnetic order parameter in the $m \Lambda_{1}\left(\rho_{1}, \rho_{2}\right)$ representation space $[20,21]$.

Considering the additional condition that the magnetic order parameter should couple to the orbital density wave (with the symmetry discussed in the previous section), allows one to make an unambiguous choice. The general

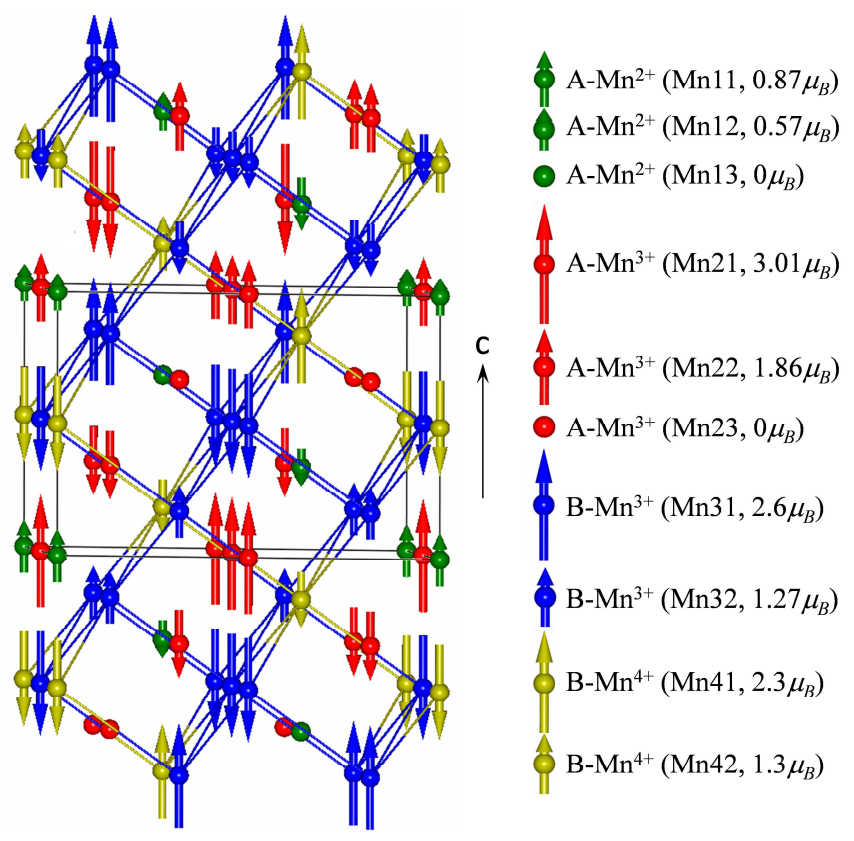

FIG. 5. Schematic representation of the commensurate longitudinal spin density wave in the high-temperature lock-in magnetic phase of $\zeta-\mathrm{Mn}_{2} \mathrm{O}_{3}$ (the values for the magnetic moments correspond to $T=55 \mathrm{~K})$.

free energy term, which couples the $\Lambda_{1}\left(\delta_{1}, \delta_{2}\right)$ structural modulation with the $m \Lambda_{1}\left(\rho_{1}, \rho_{2}\right)$ magnetic order parameters (magneto-elastic coupling), is $\delta_{1} \rho_{1}^{2}-\delta_{1} \rho_{2}^{2}+2 \delta_{2} \rho_{1} \rho_{2}$ [22]. This term indicates that between the three distinct magnetic order parameters listed in Table II, a coupling to the orbital density wave with the symmetry of $\Lambda_{1}\left(\delta_{1}, 0\right) \equiv \Lambda_{1}(\delta, 0)$ is expected only in the case of $m \Lambda_{1}\left(\rho_{1}, 0\right) \equiv m \Lambda_{1}(\rho, 0)$. Thus, the magnetic space group of the commensurate lock-in magnetic phase of $\zeta$ $\mathrm{Mn}_{2} \mathrm{O}_{3}$ is $R_{I} \overline{3}$ with the origin choice at $(0,0,0)$. This space group restricts the spin-density waves to have only cosine terms, $R_{i, k} \cos \left(2 \pi \boldsymbol{k}\left(\boldsymbol{r}_{\boldsymbol{i}}+\boldsymbol{t}\right)\right.$ ) (where $\boldsymbol{r}_{\boldsymbol{i}}$ and $\boldsymbol{t}$ are the coordinates of the $i$-th atom in a zeroth unit cell and a lattice translation of the parent structure, respectively, and $\boldsymbol{k}=\boldsymbol{k}_{\mathbf{0}}$ or $3 \boldsymbol{k}_{\mathbf{0}}$ ) and the only fitting parameters are amplitudes of the waves, $R_{i, k}$, on the four $(i=1-4)$ symmetry-independent Mn-sites in the parent structure (see Fig. 1a). The refinement resulted in a very good fitting quality (Fig. 4b). The amplitudes of the four distinct Mn-sites at $T=55 \mathrm{~K}$ are summarized in Table 3S of the Supplemental Material [23]. Magnetic moment on any atom within or outside of the parent unit cell can be obtained by the relation $R_{t o t}=R_{i, k_{0}} \cos \left(2 \pi \boldsymbol{k}_{\mathbf{0}}\left(\boldsymbol{r}_{\boldsymbol{i}}+\boldsymbol{t}\right)\right)+R_{i, 3 k_{0}} \cos \left(2 \pi 3 \boldsymbol{k}_{\mathbf{0}}\left(\boldsymbol{r}_{\boldsymbol{i}}+\boldsymbol{t}\right)\right)$.

The magnetic unit cell is eight times bigger than the nuclear cell of the parent structure and can be represented by a sequence of layers stacked along the $c$ axis with a constant moment within each layer. There are two-types of these layers for the B-site $\mathrm{Mn}^{3+} / \mathrm{Mn}^{4+}$ 
cations (Fig. 5) with the moment size 2.6(1) $\mu_{B} / 2.3(2) \mu_{B}$ (个) and $1.27(6) \mu_{B} / 1.3(1) \mu_{B}(\uparrow)$ at $T=55 \mathrm{~K}$ (see Fig. $6 \mathrm{a}$ and $\mathrm{b}$ for their temperature dependence). They correspond to the distinct Wyckoff positions in the $\left(2 \sqrt{2} a_{p} \times\right.$ $\left.2 \sqrt{2} a_{p} \times 4 \sqrt{3} a_{p}\right)$ supercell. The spins of $\mathrm{Mn}^{3+}$ and $\mathrm{Mn}^{4+}$ cations are parallel to each other in the first layer and anti-parallel in the second one. The ordering in the A-site perovskite sublattice can be obtained by stacking three types of layers with the moment size for $\mathrm{Mn}^{2+} / \mathrm{Mn}^{3+}$ being $0.81(5) \mu_{B} / 3.0(1) \mu_{B}(\uparrow), 0.57(4) \mu_{B} / 1.86(7) \mu_{B}(\uparrow)$ and zero layer $(\bullet)$, where both $\mathrm{Mn}^{2+}$ and $\mathrm{Mn}^{3+}$ do not carry any ordered moment. In the layers with non-zero moments, $\mathrm{Mn}^{2+}$ and $\mathrm{Mn}^{3+}$ spins are aligned ferrromagnetically. The translationally symmetric magnetic unit cell is obtained by repeating three blocks, each consisting of eight B-site and A-site layers stacked as $\uparrow \downarrow \uparrow \downarrow \uparrow \downarrow \uparrow$ and $\uparrow \downarrow \bullet \uparrow \downarrow \uparrow \bullet \downarrow$, respectively.

\section{Low-temperature incommensurate magnetic phase}

Below $T_{2} \sim 50 \mathrm{~K}$, a set of new magnetic reflections appears whose positions are temperature-dependent, indicating an incommensurate nature of the ground state. The available neutron diffraction data provide evidence for the presence of four distinct propagation vectors (Fig. $6 c)$. The most intense magnetic reflections can be indexed using a fundamental $\boldsymbol{k}_{\mathbf{0}}=\left(0,0, k_{z}\right)$ propagation vector, with $k_{z}$ varying as a function of temperature (Figure $4 \mathrm{a})$. The other three components can be expressed in terms of this fundamental modulation and the modulation of the orbital density wave $\boldsymbol{k}_{\boldsymbol{S}}$ (which does not change at $\left.T_{2}\right)$ as $\boldsymbol{k}_{1+}=\boldsymbol{k}_{0}+\boldsymbol{k}_{\boldsymbol{S}}, \boldsymbol{k}_{1-}=\boldsymbol{k}_{\mathbf{0}}-\boldsymbol{k}_{\boldsymbol{S}}$ and $\boldsymbol{k}_{2+}=\boldsymbol{k}_{2-}=\boldsymbol{k}_{\mathbf{0}} \pm 2 \boldsymbol{k}_{\boldsymbol{S}}$. The relations between the different modulated components of the magnetic ground state of $\zeta-\mathrm{Mn}_{2} \mathrm{O}_{3}$ are identical to those in $\mathrm{AMn}_{7} \mathrm{O}_{12}[7,11]$. In the latter case, the low-temperature incommensurate phase is a helix whose helicity is modulated with the periodicity of the orbital density wave. Attempts to adapt a similar helical structure for $\zeta-\mathrm{Mn}_{2} \mathrm{O}_{3}$ did not result in a satisfactory fitting. Moreover, this magnetic structure is not compatible with the high-temperature longitudinal spin-density wave. The latter implies uniaxial anisotropy, whereas a helix usually takes place in systems with easyplane anisotropy. A logical compromise between the uniaxial anisotropy and a moment-conserving ground state, expected for an insulator, is a cycloidal ordering. The method used to construct the phase-modulated helix for $\mathrm{CaMn}_{7} \mathrm{O}_{12}$ [7] and other members of the $\mathrm{AMn}_{7} \mathrm{O}_{12}$ family [11] can be used to obtain a phase-modulated cycloidal structure. In this case, the fundamental component $\boldsymbol{k}_{\mathbf{0}}$ is combined with additional cycloids $\boldsymbol{k}_{\mathbf{1}} / \boldsymbol{k}_{\mathbf{1}}$ and $\boldsymbol{k}_{2+} / \boldsymbol{k}_{\mathbf{2}}$ - whose magnetic phases are different from $\boldsymbol{k}_{\mathbf{0}}$ by $\pi / 2$ and $\pi$, respectively. In addition, to keep the moment constant, their amplitudes should be related by
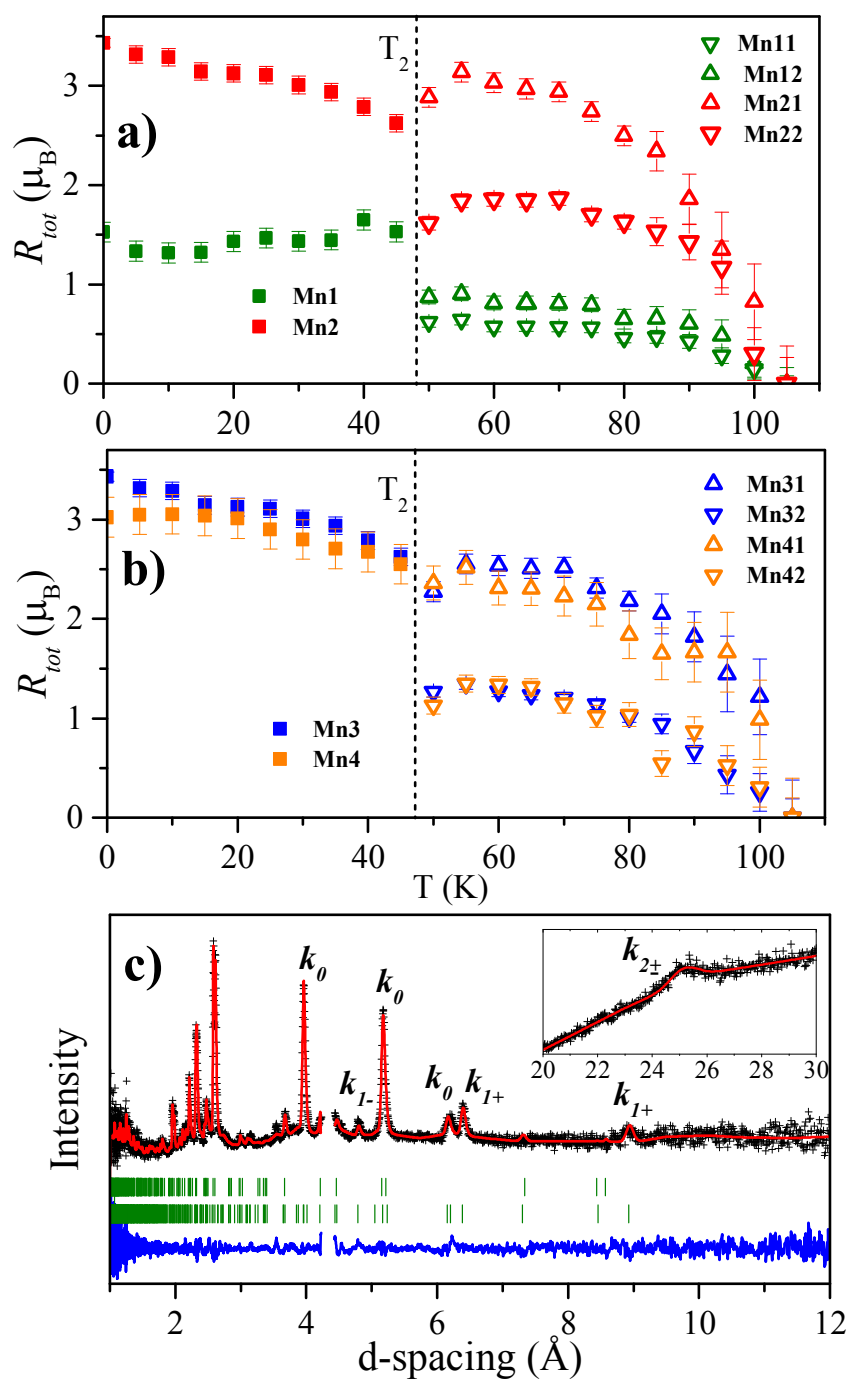

FIG. 6. (a) and (b) Mn ordered moments as a function of temperature (see Fig. 1b for notations of the Mn sites). (c) Rietveld refinement of the neutron diffraction pattern collected at $T=10 \mathrm{~K}$. The cross symbols (black) and solid line (red) represent the experimental and calculated intensities, respectively, and the line below (blue) is the difference between them. Tick marks indicate the positions of Bragg peaks for the nuclear (top), vanadium can (middle), and magnetic (bottom) scattering. The insert shows a magnified portion of the diffraction pattern, where a satellite due to the $\boldsymbol{k 2} \pm$ propagation vector is observed.

$R_{k 2}=R_{k 1}^{2} /\left(2 R_{k 0}\right)$, where $R_{k 0}, R_{k 1-}=R_{k 1+} \equiv R_{k 1}$, and $R_{k 2-}=R_{k 2+} \equiv R_{k 2}$ are amplitudes of the $\boldsymbol{k}_{\mathbf{0}}, \boldsymbol{k}_{\mathbf{1} \pm}$, and $k_{2} \pm$ components, respectively. The total moment size of the phase-modulated cycloid is $R_{t o t}=R_{k 0}+2 R_{k 2}$. This model was applied to refine the ground state of $\zeta-\mathrm{Mn}_{2} \mathrm{O}_{3}$ and worked reasonably well, but the refinement could be further improved by a tilting of the spin plane by 20(2) degrees out from the c-axis. This implies an admixture of a helical component, which points to the presence of an additional anisotropic term competing with the uniax- 
ial anisotropy. The obtained general magnetic structure (Fig. 7a), involving both the major cycloidal and the minor helical components, provides a good fitting quality (Fig. 6c) with the parameters summarized in Table $4 \mathrm{~S}$ of the Supplemental Material [23]. In the refinement procedure, only the Fourier coefficients related to the $\boldsymbol{k}_{\mathbf{0}}$ and $\boldsymbol{k}_{1 \pm}$ cycloidal/helical components were actually refined, the coefficients of the $\boldsymbol{k}_{\mathbf{2}} \pm$ components were fixed through the $R_{k 2}=R_{k 1}^{2} /\left(2 R_{k 2}\right)$ relation, to keep a constant moment size in the phase-modulated structure. The ordered moments $\left(R_{\text {tot }}\right)$, for the four symmetry-independent Mn sites of the parent structure, as functions of temperature are plotted in Figure $6 \mathrm{a}$ and b. The saturated spins of $\mathrm{Mn}^{3+}$ (Mn2 and Mn3 sites) and $\mathrm{Mn}^{2+}$ (Mn1 site) are reduced in comparison with their theoretical values. This reduction is most pronounced in the latter case, where the refined value is only $\sim 1.53(8) \mu_{B}$ instead of the expected $5 \mu_{B}$ for the high-spin configuration of a $d^{5}$ ion.

The magnetic point group of the phase modulated cycloid is polar $1^{\prime}$, implying the presence of an improper ferroelectric polarization below $T_{2}$ (note the point group of the high-temperature phase is non-polar $\overline{3} 1^{\prime}$ ). Thus, like other members of the $\mathrm{AMn}_{7} \mathrm{O}_{12}$ family, $\zeta-\mathrm{Mn}_{2} \mathrm{O}_{3}$ is a type-II multiferroic, whose multiferroic properties have indeed been recently confirmed by dielectric and pyroelectric measurements [15].

\section{DISCUSSION}

Let us show that the complex magnetic ground state involving several distinct magnetic order parameters $\boldsymbol{k}_{\boldsymbol{n}} \pm$ and the high-temperature commensurate structure of $\zeta$ $\mathrm{Mn}_{2} \mathrm{O}_{3}$ can be qualitatively understood as a coupling phenomenon based on a simple phenomenological approach developed to explain the lock-in phase and multi$k$ helical structure of $\mathrm{CaMn}_{7} \mathrm{O}_{12}$ [7]. In the Supplemental Material [23] we briefly comment on an alternative scenario to describe the lock-in phase in $\mathrm{CaMn}_{7} \mathrm{O}_{12}$, proposed by Sławiński et al [28], using magnetic superspace group formalism. The phenomenological freeenergy invariants for the ground state helical component of $\zeta-\mathrm{Mn}_{2} \mathrm{O}_{3}$ are identical to the case of $\mathrm{CaMn}_{7} \mathrm{O}_{12}$ [7], but the approach requires some modification for the cycloidal order. To find the explicit form of the coupling terms related to the cycloid, we can use the matrix operators defined on a complex basis and provided in Table 1 of the Supplementary Material of Ref. [7]. In this case, however, both time-even (for structural modulation) and time-odd (for magnetic order) $\Lambda_{1}$ representations are relevant, so we specify the corresponding complex order parameters as $\Lambda_{1}\left(\delta, \delta^{*}\right)$ and $m \Lambda_{1}(\rho, \rho *)$, respectively. A spin cycloid with a $\boldsymbol{k}_{\boldsymbol{n}} \pm$ propagation vector is formed by a combination of $m \Lambda_{2} \oplus \Lambda_{3}\left(\eta_{n \pm}, \xi_{n \pm}^{*}, \xi_{n \pm}, \eta_{n \pm}^{*}\right)$ and $m \Lambda_{1}\left(\rho_{n \pm}, \rho_{n \pm}^{*}\right)$ order parameters, taken with real and imaginary characters,
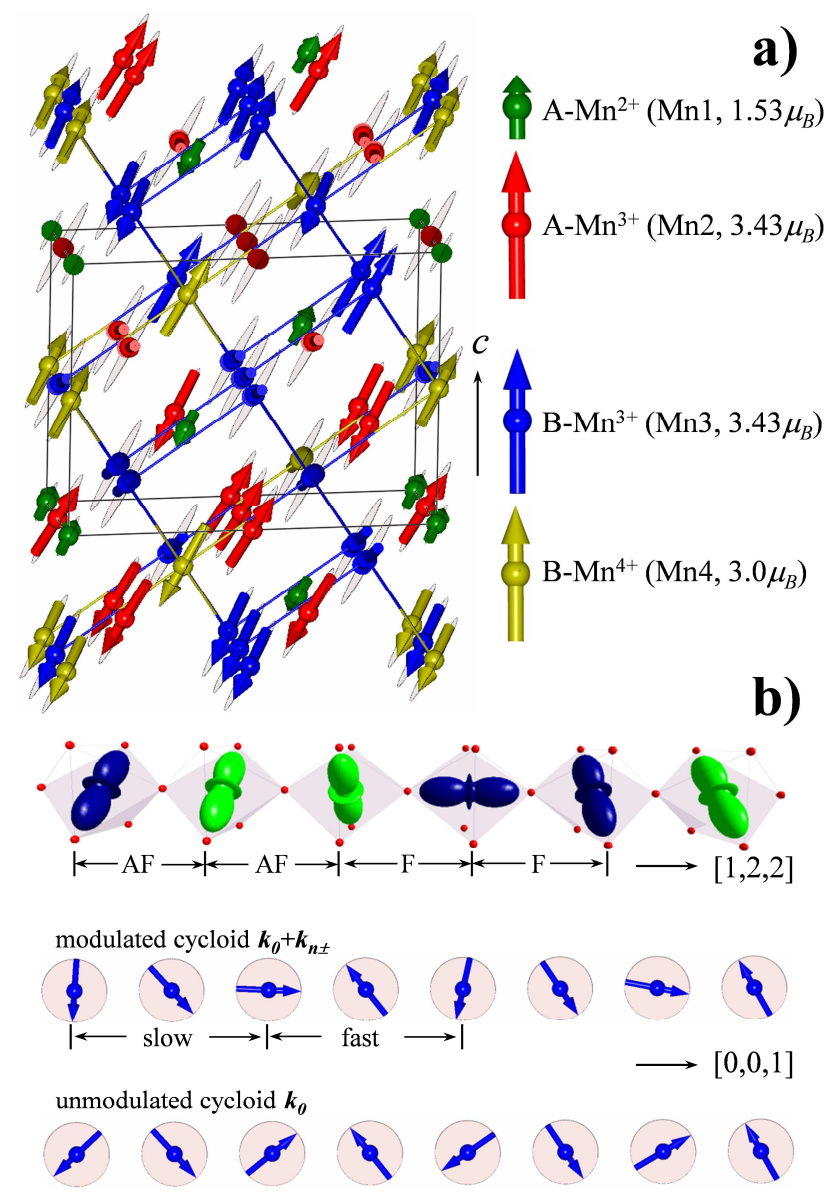

FIG. 7. (a) Schematic representation of the magnetic structure in the low-temperature incommensurate phase of $\zeta$ $\mathrm{Mn}_{2} \mathrm{O}_{3}$ (the values for the magnetic moments correspond to $T=10 \mathrm{~K}$ ). (b) A portion of the orbital density wave, demonstrating the modulation of the 180-degrees superexchange interaction between the neighbouring $\mathrm{B}$-site $\mathrm{Mn}^{3+}$ cations along the $[1,2,2]$ direction (in the setting of parent structure). Below, fragments of the cycloidal component of the magnetic ground state with modulated and unmodulated spin chirality are shown.

respectively (implying the phase shift by $\pi / 2$ ). The free-energy coupling terms responsible for the admixture of the $\boldsymbol{k}_{\boldsymbol{n}+}$ and $\boldsymbol{k}_{\boldsymbol{n}}$ - components are: $\left(\delta_{S}^{n} \xi_{0} \xi_{n+}^{*}+\right.$ $\left.\delta_{S}^{* n} \xi_{0}^{*} \xi_{n+}+\delta_{S}^{n} \eta_{0} \eta_{n+}^{*}+\delta_{S}^{* n} \eta_{0}^{*} \eta_{n+}\right)+\left(\delta_{S}^{n} \rho_{0} \rho_{n+}^{*}+\delta_{S}^{* n} \rho_{0}^{*} \rho_{n+}\right)$ and $\left(\delta_{S}^{n} \xi_{0}^{*} \xi_{n+}+\delta_{S}^{* n} \xi_{0} \xi_{n+}^{*}+\delta_{S}^{n} \eta_{0}^{*} \eta_{n+}+\delta_{S}^{* n} \eta_{0} \eta_{n+}^{*}\right)+$ $\left(\delta_{S}^{n} \rho_{0}^{*} \rho_{n+}+\delta_{S}^{* n} \rho_{0} \rho_{n+}^{*}\right)$, respectively. At a microscopic level, these energy terms represent a modulation of exchange interactions and magnetic anisotropy by the orbital density wave. To demonstrate this effect, fragments of the modulated and unmodulated cycloids are shown in Figure $7 \mathrm{~b}$ (only spins on the B-site $\mathrm{Mn}^{3+}$ are shown for clarity). One can see the regions where the spins rotate slower and faster upon propagation through the cycloid, in comparison with the unmodulated structure. The periodicity of the modulation of the spin rotation (spin chirality) is the same as the periodicity of the struc- 
tural modulation $\boldsymbol{k}_{\boldsymbol{S}}$. A fragment of the orbital density wave shown for several corner-sharing octahedra coordinating the $\mathrm{B}$-site $\mathrm{Mn}^{3+}$, provides a clear demonstration as to how the orbital ordering modulates the exchange interactions between the B-site cations. Following the qualitative Goodenough-Kanamori rules for 180-degree superexchange [27], one should expect a dominance of ferromagnetic interactions in the regions marked as $\mathrm{F}$ and antiferromagnetic interactions in the AF regions. Although, the cycloidal plane does not contain the [122] direction of the corner-sharing octahedra, the slow and fast spin rotations in the cycloid is the direct consequence of this modulation of the exchange interactions. The modulation of the spin chirality is rather strong in $\zeta-\mathrm{Mn}_{2} \mathrm{O}_{3}$, apparently due to the fact that the commensurate orbital density wave involves stacking of almost fully polarized orbital states.

The magneto-elastic term that describes the coupling between the fundamental components $\boldsymbol{k}_{\mathbf{0}}$ and the orbital density wave $\boldsymbol{k}_{\boldsymbol{S}}$, is given by: $\left(\delta_{S} \xi_{0}^{*} \eta_{0}^{*}+\delta_{S}^{*} \xi_{0} \eta_{0}\right)-\left(\delta_{S}^{*} \rho_{0}^{2}+\right.$ $\left.\delta_{S} \rho_{0}^{2}\right)$. Note, the values of the $m \Lambda_{2} \oplus \Lambda_{3}\left(\eta_{0}, \xi_{0}^{*}, \xi_{0}, \eta_{0}^{*}\right)$ and $m \Lambda_{1}\left(\rho_{0}, \rho_{0}^{*}\right)$ order parameters are not required by symmetry to be equal (in the magnetic structure refinements, however, this constraint was imposed to reduce the number of fitting parameters without compromising the reliability of the fit). The translational invariance of this term requires the relation $\boldsymbol{k}_{\boldsymbol{S}}-2 \boldsymbol{k}_{0}=(0,0,3) n(n=1$ in the high-temperature lock-in phase) and the coupling is most efficient for the case of a spin-density wave (when either $m \Lambda_{2} \oplus \Lambda_{3}\left(\eta_{0}, \xi_{0}^{*}, \xi_{0}, \eta_{0}^{*}\right)$ or $m \Lambda_{1}\left(\rho_{0}, \rho_{0}^{*}\right)$ vanishes $)$. An admixture of both order parameters (cycloidal structure) reduces the coupling, and the term takes its minimal value when the amplitudes of both spin density waves $m \Lambda_{2} \oplus \Lambda_{3}\left(\eta_{0}, \xi_{0}^{*}, \xi_{0}, \eta_{0}^{*}\right)$ and $m \Lambda_{1}\left(\rho_{0}, \rho_{0}^{*}\right)$ are maximal (when the spins are saturated). This is due to the fact that the phase shift between $m \Lambda_{2} \oplus \Lambda_{3}\left(\eta_{0}, \xi_{0}^{*}, \xi_{0}, \eta_{0}^{*}\right)$ and $m \Lambda_{1}\left(\rho_{0}, \rho_{0}^{*}\right)$ is $\pi / 2$, which implies that the corresponding $\Lambda_{1}\left(\delta_{S}, \delta_{S}^{*}\right)$ order parameters (structural modulations) coupled to them are in anti-phase. This provides a natural explanation why the low-temperature magnetic structure de-locks from the structural modulation, giving rise to the incommensurate ground state. The magnetic structure with saturated moments is favoured by entropy, but the vanishingly small magneto-elastic coupling of this phase is not sufficient to hold the lock-in. As a result, the system de-locks and selects the magnetic propagation vector that minimises the competing exchange interactions (see Refs. $[1,11]$ for discussion of the exchange interactions resulting in an incommensurate magnetic propagation vector in $\mathrm{AMn}_{7} \mathrm{O}_{12}$ manganites).

\section{CONCLUSIONS}

The metastable perovskite modification of $\zeta-\mathrm{Mn}_{2} \mathrm{O}_{3}$ stabilized under high-pressure and high-temperature con- ditions exhibits the 3:1 charge ordering between the B-site $\mathrm{Mn}^{3+}$ and $\mathrm{Mn}^{4+}$ cations, and a unique orbital texture involving the orbital states alternating in a $\Psi^{+} \Psi^{+} \Psi^{-} \Psi^{-}$fashion (+ and - indicate distinct orbital states). The orbital pattern localised on the B-site $\mathrm{Mn}^{3+}$ is a commensurate limit of the orbital density wave observed in $\mathrm{CaMn}_{7} \mathrm{O}_{12}$. The orbital wave propagation vector in $\zeta-\mathrm{Mn}_{2} \mathrm{O}_{3}$ is $\boldsymbol{k}_{\boldsymbol{S}}=(0,0,3 / 4)$, specified in respect of the parent $R \overline{3}\left(2 \sqrt{2} a_{p} \times 2 \sqrt{2} a_{p} \times \sqrt{3} a_{p}\right)$ structure, and the global phase of the associated structural modulation is $\phi_{0}=0$. The commensurate value of the propagation vector and the choice of the global phase are imposed by a coupling of the orbital modulation to independent structural distortions, with the propagation vector $\boldsymbol{k}_{\boldsymbol{T}}=(0,0,3 / 2)$, that serve to improve poor bonding conditions of the A-site $\mathrm{Mn}^{2+}$ cations.

Long-range magnetic order in the form of an anharmonic longitudinal spin density wave with the commensurate propagation vector $\boldsymbol{k}_{\mathbf{0}}=(0,0,9 / 8)$ onsets below $T_{1} \sim 100 \mathrm{~K}$. The propagation vector is locked to the structural modulation through the relation $\boldsymbol{k}_{S}+2 \boldsymbol{k}_{0}=$ $(0,0,3)$, imposed by magneto-elastic coupling that is maximal for a spin density wave-type of ordering. The magnetic structure is only partially ordered and contains $1 / 4$ of the A-site Mn cations with zero ordered moment. This lock-in magnetic phase is stable down to $T_{2} \sim 50 \mathrm{~K}$. Below this temperature, the periodicity of the magnetic subsystem de-locks from that of the structural modulation, resulting in a nearly constant moment incommensurate ground state, which combines both phase-modulated cycloidal and helical components. The spin chirality in the ground state magnetic structure is modulated with the periodicity of the commensurate orbital density wave. This remarkable phenomenon is a result of a magnetoorbital coupling mechanism that is common to the whole series of $\mathrm{A}^{2+} \mathrm{Mn}_{7} \mathrm{O}_{12}\left(\mathrm{~A}^{2+}=\mathrm{Mn}, \mathrm{Ca}, \mathrm{Sr}, \mathrm{Pb}\right.$ and $\left.\mathrm{Cd}\right)$ quadruple manganites studied to date.

\section{ACKNOWLEDGMENTS}

The work done at ISIS was supported by the project TUMOCS. This project has received funding from the European Union's Horizon 2020 research and innovation programme under the Marie Skłodowska-Curie grant agreement No 645660. RDJ acknowledges support from a Royal Society University Research Fellowship.

* email: dmitry.khalyavin@stfc.ac.uk

[1] N. Perks, R. Johnson, C. Martin, L. Chapon, and P. Radaelli, Nat. Commun. 3, 1277 (2012).

[2] R. D. Johnson, L. C. Chapon, D. D. Khalyavin, P. Manuel, P. G. Radaelli, and C. Martin, Phys. Rev. Lett. 108, 067201 (2012). 
[3] R. Przenioslo, I. Sosnowska, E. Suard, A. Hewat, and A. N. Fitch, J. Phys.: Condens. Matter 14, 5747 (2002).

[4] R. Przenioslo, I. Sosnowska, E. Suard, A. Hewat A. N. Fitch, Physica B, 344, 358 (2004).

[5] W. Slawinski, R. Przenioslo, I. Sosnowska, M. Bieringer, I. Margiolaki, and E. Suard, Acta Crystallogr., Sect. B: Struct. Sci. 65, 535 (2009).

[6] W. Slawinski, R. Przenioslo, I. Sosnowska, and M. Bieringer, J. Phys.: Condens. Matter 22, 186001 (2010).

[7] R. D. Johnson, D. D. Khalyavin, P. Manuel, A. Bombardi, C. Martin, L. C. Chapon, and P. G. Radaelli, Phys. Rev. B 93, 180403(R) (2016).

[8] W. Slawinski, R. Przenioslo, I. Sosnowska, and A. Chrobak, J. Phys. Soc. Jpn. 81, 094708 (2012).

[9] N. Terada, Y. S. Glazkova, and A. A. Belik, Phys. Rev. B 93, 155127 (2016).

[10] A. A. Belik, Y. S. Glazkova, N. Terada, Y. Matsushita, A. V. Sobolev, I. A. Presniakov, N. Tsujii, S. Nimori, K. Takehana, and Y. Imanaka, Inorg. Chem. 55, 6169 (2016).

[11] R. D. Johnson, D. D. Khalyavin, P. Manuel, P. G. Radaelli, I. S. Glazkova, N. Terada, and A. A. Belik, Phys. Rev. B 96, 054448 (2017).

[12] Yana S. Glazkova, Noriki Terada, Yoshitaka Matsushita, Yoshio Katsuya, Masahiko Tanaka, Alexey V. Sobolev, Igor A. Presniakov, and Alexei A. Belik, Inorg. Chem. 54, 9081 (2015).

[13] A. A. Belik, Y. S. Glazkova, Y. Katsuya, M. Tanaka, A. V. Sobolev, and I. A. Presniakov, J. Phys. Chem. C 120, 8278 (2016).

[14] S. V. Ovsyannikov, A. M. Abakumov, A. A. Tsirlin, W. Schnelle, R. Egoavil, J. Verbeeck, G. Van Tendeloo, K. V. Glazyrin, M. Hanfland, and L. Dubrovinsky, Angew. Chem. Int. Ed. 52, 1494 (2013).

[15] J. Cong, K. Zhai, Y. Chai, D. Shang, D. D. Khalyavin, R. D. Johnson, D. P. Kozlenko, S. E. Kichanov, A. M. Abakumov, A. A. Tsirlin, L. Dubrovinsky, X. Xu, Z. Sheng, S. V. Ovsyannikov, and Y. Sun, submitted to
Nat. Commun.

[16] S. V. Ovsyannikov, A. E. Karkin, N. V. Morozova, V. V. Shchennikov, E. Bykova, A. M. Abakumov, A. A. Tsirlin, K. V. Glazyrin, and L. Dubrovinsky, Adv. Mater. 26, 8185 (2014).

[17] L. C. Chapon, P. Manuel, P. G. Radaelli, C. Benson, L. Perrott, S. Ansell, N. J. Rhodes, D. Raspino, D. Duxbury, E. Spill, J. Norris, Neutron News 22, 22 (2011).

[18] V. L. Aksenov, A. M. Balagurov, V. P. Glazkov, D. P. Kozlenko, I. V. Naumov, B. N. Savenko, D. V. Sheptyakov, V. A. Somenkov, A. P. Bulkin, V. A. Kudryashev and V. A. Trounov, Materials Science Forum 321-324 326 (2000).

[19] J. Rodriguez Carvajal, Physica B 193, 55 (1993).

[20] H. T. Stokes, D. M. Hatch, and B. J. Campbell, ISOTROPY Software Suite, iso.byu.edu.

[21] B. J. Campbell, H. T. Stokes, D. E. Tanner, and D. M. Hatch, J. Appl. Crystallogr. 39, 607 (2006).

[22] To construct the free-energy invariant, we adopt the real irreps matrices implemented in ISOTROPY [20] and ISODISTORT [21] software (irrep version 2011) and used to generate the isotropy subgroups.

[23] See Supplemental Material at [] for results of the crystal and magnetic structure refinemnts of $\zeta-\mathrm{Mn}_{2} \mathrm{O}_{3}$.

[24] A. A. Belik, Y. Matsushita, D. D. Khalyavin, Angew Chem Int Ed Engl. 56, 10423 (2017).

[25] B. N. Figgis, M. A. Hitchman, Ligand-Field Theory and Its Applications, Wiley-VCH, New York, 2000.

[26] All the magnetic reflections in the high-temperature commensurate phase can be indexed using a single propagation vector $\boldsymbol{k}=(0,0,3 / 2)$ and the large $\left(2 \sqrt{2} a_{p} \times 2 \sqrt{2} a_{p} \times\right.$ $\left.4 \sqrt{3} a_{p}\right)$ supercell. This approach to solve the magnetic structure has, however, a serious disadvantage since it implies a presence of many nominally independent $\mathrm{Mn}$ sites in the supercell.

[27] J. B. Goodenough, Magnetism And The Chemical Bond (Interscience Publishers, New York, London, 1963).

[28] W. Slawinski, R. Przenioslo, I. Sosnowska and V. Petricek, Acta Cryst. B68, 240 (2012). 